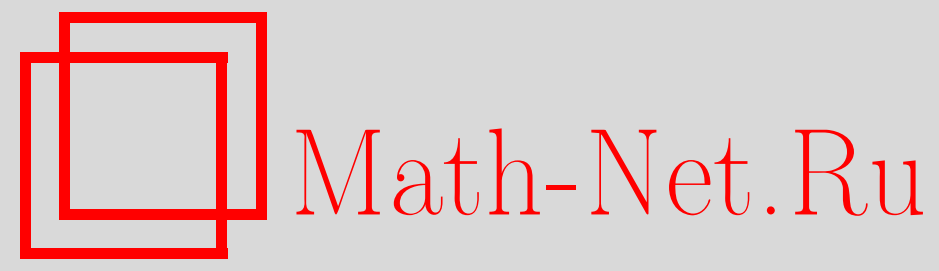

И. Ю. Денисова, Асимптотическое поведение решения системы стохастических дифференциальных уравнений, Теория вероятн. и ее примен., 1996, том 41, выпуск 2, 425-429

DOI: https://doi.org/10.4213/tvp2955

Использование Общероссийского математического портала Math-Net.Ru подразумевает, что вы прочитали и согласны с пользовательским соглашением http://www . mathnet.ru/rus/agreement

Параметры загрузки:

IP : 18.234 .156 .22

26 апреля 2023 г., 16:48:55

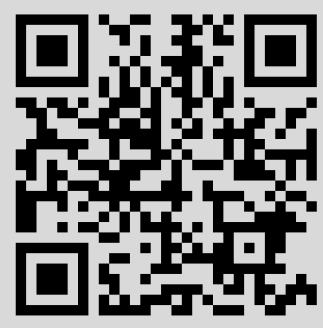


5. Горяймов В. В. Дробное итерирование вероятностных производящих функций и вложение дискретных ветвящихся процессов в непрерывные. - Матем. сб., 1993, т. 184, № 5 , c. 55-74.

(c) $1996 \mathrm{r}$.

ДЕНИСОВА И. ЮО.*

\section{АСИМПТОТИЧЕСКОЕ ПОВЕДЕНИЕ РЕШЕНИЯ СИСТЕМЫ СТОХАСТИЧЕСКИХ ДИФФЕРЕНЦИАЛЬНЫХ УРАВНЕНИЙ}

В работе изучается асимптотическое поведение угловой компоненты решения двумерной системы нелинейных стохастических дифференциальных уравнений специального вида в некоторой области. Эта область представляет собой кольцо, внешня и внутренняя окружности которого являются инвариантными множествами соответствуюшей линеаризированной системы.

Ключевые слова и Фразы: стохастическое дифференциальное уравнение Ито, инвариантное множество, стационарное множество, предел с вероятностью единица.

Пусть существует решение $\zeta(t)=\left(\zeta_{1}(t), \ldots, \zeta_{n}(t)\right)$ системы стохастических дифференциальных уравнений

$$
d \zeta(t)=a(\zeta(t)) d t+b(\zeta(t)) d w(t)
$$

где $t \geqslant 0, a(x)=\left(a_{1}(x), \ldots, a_{n}(x)\right), b(x)=\left(b_{1}(x), \ldots, b_{n}(x)\right)$ - действительные измеримые функции, $x=\left(x_{1}, \ldots, x_{n}\right)$ - точка из $\mathbf{R}^{n}, w(t)$ - одномерный винеровский процесс, заданный на вероятностном пространстве $(\Omega, \mathcal{F}, P)$. Пусть решение $\zeta(t)$ при $t=0$ принимает значение $x^{0}=\left(x_{1}^{0}, \ldots, x_{n}^{0}\right), x^{0}$ - неслучайное, а коэффициенты $a(x)$ и $b(x)$ допускают разложения

$$
a(x)=A x+\widetilde{a}(x), \quad b(x)=B x+\widetilde{b}(x) .
$$

Рассмотрим систему линейных стохастических дифференциальных уравнений, соответствуюшую данной нелинейной системе (1):

$$
d \xi(t)=A \xi(t) d t+B \xi(t) d w(t) .
$$

Пусть $D$ - открытая область в $\mathbf{R}^{n}$ и $\tau_{D}$ - момент первого выхода процесса $\xi(t)$ из области $D$.

O п р е д е л е и и е 1 . Непустое множество $\Gamma \subseteq D$ называется инвариантным множеством системы (2) до момента первого выхода $\tau_{D}$, если

$$
\mathbf{P}\left\{\xi(t) \in \Gamma \forall t<\tau_{D} \mid \xi(0) \in \Gamma\right\}=1 .
$$

Пусть уравнение инвариантного множества системы (2) имеет вид $G(x)=C$, где $C$ - некоторая постоянная, функция $G(x) \in C^{2}(D)[1]$.

*Московский институт радиотехники, электроники и автоматики, просп. Вернадского, 78, Москва, Россия. 
О п р е д е л е н и е 2. Множество $\{x: G(x)=C\}$ называется стационарным для системы уравнений (1), если оно является инвариантным множеством соответствующей линеаризированной системы (2) и

$$
\left.\widetilde{a}(x)\right|_{G(x)=C}=0,\left.\quad \tilde{b}(x)\right|_{G(x)=C}=0 .
$$

Заметим, что если $x^{0}$ находится на стационарном множестве, то $\zeta(t)=C$ для всех $t>0$, а если $x^{0} \in\{G(x)>C\}$, то выполняется $\zeta(t)>C$ для всех $t>0$ [2].

Пусть размерность пространства $n=2$, и матрицы $A$ и $B$ имеют вид

$$
B=\left(\begin{array}{cc}
0 & \mu \\
-\mu & 0
\end{array}\right), \quad A=\left(\begin{array}{cc}
-\frac{\mu^{2}}{2} & m \mu \\
-m \mu & -\frac{\mu^{2}}{2}
\end{array}\right),
$$

где $\mu=$ const $>0, m-$ произвольная постоянная.

Тогда уравнения инвариантных множеств системы (2) имеют вид $x_{1}^{2}+x_{2}^{2}=C$, где $C$ - произвольная неотрицательная постоянная [1]. Перейдем от декартовых координат $x_{1}, x_{2}$ к полярным $r, \varphi: x_{1}=r \cos \varphi, x_{2}=r \sin \varphi$. Разобьем процесс $\zeta(t)$ на две составляющие: радиальную $r(t)$ и угловую $\varphi(t)$. Обозначим

$$
\begin{array}{rlrl}
\lambda & =(\cos \varphi, \sin \varphi), \quad \lambda^{\perp}=(-\sin \varphi, \cos \varphi), \\
x & =\left(x_{1}, x_{2}\right), \quad x^{\perp}=\left(-x_{2}, x_{1}\right), & b(r, \varphi)=\tilde{b}(r \cos \varphi, r \sin \varphi), \\
\bar{a}(r, \varphi) & =\tilde{a}(r \cos \varphi, r \sin \varphi), \quad \sigma(r, \varphi)=\frac{1}{r}\left(\bar{b}(r, \varphi), \lambda^{\perp}\right) .
\end{array}
$$

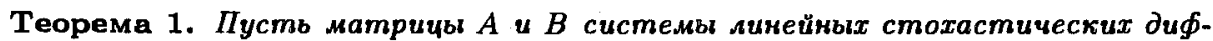
Ференциальных уравнекий (2) имеют вид (3) и множества $\left\{x: x_{1}^{2}+x_{2}^{2}=C_{i}, i=1,2\right\}$ являяотся стационарными для системы (1).

1. $\operatorname{Ecsu}\left(\bar{b}(r, \varphi), \lambda^{\perp}\right)=0,\left(\bar{a}(r, \varphi), \lambda^{\perp}\right)+\mu\left(\bar{b}(r, \varphi), \lambda^{\perp}\right)=0$, mo npu $m \neq 0 \quad c$ веровтностья единииа $\varphi(t) / t \rightarrow-m \mu$ при $t \rightarrow \infty$. При $m=0$

$$
\mathbf{P}\left\{\frac{\varphi(t)}{\sqrt{t}}<y\right\} \underset{t \rightarrow \infty}{\longrightarrow} \frac{1}{\sqrt{2 \pi}|\mu|} \int_{-\infty}^{y} \exp \left\{-\frac{u^{2}}{2 \mu^{2}}\right\} d u .
$$

2. Ecsu $m \mu \neq 0,0<-\mu+(1 / r)\left(\vec{b}(r, \varphi), \lambda^{\perp}\right) \leqslant c$,

$$
\left(\bar{a}(r, \varphi), \lambda^{\perp}\right)+\mu(\bar{b}(r, \varphi), \lambda)-\frac{1}{r}(\bar{b}(r, \varphi), \lambda)\left(\bar{b}(r, \varphi), \lambda^{\perp}\right)=0
$$

mo

$$
\mathbf{P}\left\{\lim _{t \rightarrow \infty} \frac{\varphi(t)}{t}=-m \mu\right\}=1
$$

3. Ecsu

$$
\begin{gathered}
-m \mu+\frac{1}{r}\left(\left(\bar{a}(r, \varphi), \lambda^{\perp}\right)+\mu(\bar{b}(r, \varphi), \lambda)\right)-\frac{1}{r}(\bar{b}(r, \varphi), \lambda)\left(\bar{b}(r, \varphi), \lambda^{\perp}\right)=\widehat{a}(\varphi), \\
0<-\mu+\sigma(r, \varphi)=\widehat{\sigma}(\varphi), \quad\left|\int_{0}^{t} \frac{\widehat{a}(v)}{\widehat{\sigma}^{2}(v)} d v\right|<c_{1}
\end{gathered}
$$

$u$

$$
\begin{aligned}
& \frac{1}{\varphi} \int_{0}^{\varphi} \exp \left\{\frac{\widehat{a}(v)}{\widehat{\sigma}^{2}(v)} d v\right\} d u \longrightarrow k_{1}, \quad|\varphi| \rightarrow \infty, \\
& \frac{1}{\varphi} \int_{0}^{\varphi} \exp \left\{2 \int_{0}^{u} \frac{\widehat{a}(v)}{\widehat{\sigma}^{2}(v)} d v\right\} \frac{1}{\widehat{\sigma}^{2}(u)} d u \longrightarrow k_{2}, \quad|\varphi| \rightarrow \infty
\end{aligned}
$$


Aсимптотическое поведение решекия

427

$0<k_{i}<\infty, i=1,2, m o$

$$
\mathbf{P}\left\{\frac{\varphi(t)}{\sqrt{t}}<y\right\} \underset{t \rightarrow \infty}{\longrightarrow} \sqrt{\frac{k_{1} k_{2}}{2 \pi}} \int_{-\infty}^{y} \exp \left\{-\frac{u^{2} k_{1} k_{2}}{2}\right\} d u
$$

Д ока з а т е ль с т в о. Возьмем функцию $f\left(x_{1}, x_{2}\right)=x_{1} / \sqrt{x_{1}^{2}+x_{2}^{2}}$. Поскольку в полярных координатах $\zeta_{1}(t)=r(t) \cos \varphi(t), \zeta_{2}(t)=r(t) \sin \varphi(t)$, то $\cos \varphi(t)=$ $\zeta_{1}(t) / \sqrt{\zeta_{1}^{2}(t)+\zeta_{2}^{2}(t)}$. Построим длх процесса $\varphi(t)$ уравюнене следующим образом. Пусть искомое уравнение имеет вид $d \varphi(t)=\alpha(t) d t+\beta(t) d w(t)$, где $\alpha(t)$ и $\beta(t)-$ некоторые функции. Тогда процесс $\cos \varphi(t)$ имеет дифференциал

$$
d \cos \varphi(t)=\left(-\sin \varphi(t) \alpha(t)-\frac{1}{2} \cos \varphi(t) \beta^{2}(t)\right) d t-\sin \varphi(t) \beta(t) d w(t) .
$$

Используя формулу Ито для процесса $f\left(\zeta_{1}(t), \zeta_{2}(t)\right)$ и сравнивая члены, стоящие при $d t$ и $d w(t)$, получим

$$
\begin{aligned}
& \alpha(t)=\frac{\left(a^{*}(r(t), \varphi(t)), \lambda^{\perp}\right)}{r(t)}-\frac{\left(b^{*}(r(t), \varphi(t)), \lambda\right)\left(b^{*}(r(t), \varphi(t)), \lambda^{\perp}\right)}{r^{2}(t)}, \\
& \beta(t)=\frac{\left(b^{*}(r(t), \varphi(t)), \lambda^{\perp}\right)}{r(t)}
\end{aligned}
$$

где $a^{*}(r, \varphi)=a(r \cos \varphi, r \sin \varphi), b^{*}(r, \varphi)=b(r \cos \varphi, r \sin \varphi)$. Учитывая вид матриц $A$ и $B$, получаем уравнение для процесса $\varphi(t)$ :

$$
\begin{aligned}
\varphi(t)=\varphi(0)+\int_{0}^{t}\{ & -m \mu+\frac{1}{r(s)}\left[\left(\bar{a}(r(s), \varphi(s)), \lambda^{\perp}\right)+\mu(\bar{b}(r(s), \varphi(s)), \lambda)\right] \\
& \left.-\frac{1}{r(s)}(\bar{b}(r(s), \varphi(s)), \lambda)\left(\bar{b}(r(s), \varphi(s)), \lambda^{\perp}\right)\right\} d s \\
+ & \int_{0}^{t}\left(-\mu+\frac{1}{r}\left(\bar{b}(r(s), \varphi(s)), \lambda^{\perp}\right)\right) d w(s) .
\end{aligned}
$$

Обозначим

$$
f_{1}(r, \varphi)=\frac{1}{r}\left[\left(\bar{a}(r, \varphi), \lambda^{\perp}\right)+\mu(\bar{b}(r, \varphi), \lambda)\right]-\frac{1}{r}(\bar{b}(r, \varphi), \lambda)\left(\bar{b}(r, \varphi), \lambda^{\perp}\right) .
$$

Если $\left(\bar{b}(r, \varphi), \lambda^{\perp}\right)=0,(\bar{a}(r, \varphi), \lambda)+\mu(\bar{b}(r, \varphi), \lambda)=0$, то уравнение для $\varphi(t)$ имеет вид $\varphi(t)=\varphi(0)-m \mu t-\mu w(t)$. Так как с вероятностью единица $w(t) / \sqrt{t} \longrightarrow 0$ при $t \rightarrow \infty$, то с вероятностьо единица $\varphi(t) / t \rightarrow-m \mu$ при $t \rightarrow \infty$. При $m=0$, поскольку $w(t) / \sqrt{t}$ имеет нормальное распределение с параметрами $(0,1)$, то

$$
\lim _{t \rightarrow \infty} \mathbf{P}\left\{\frac{\varphi(t)}{\sqrt{t}}<y\right\}=\frac{1}{\sqrt{2 \pi}|\mu|} \int_{-\infty}^{y} \exp \left\{-\frac{u^{2}}{2 \mu^{2}}\right\} d u .
$$

Если $m \mu \neq 0,0<-\mu+\sigma(r, \varphi)<c$,

$$
\left(\bar{a}(r, \varphi), \lambda^{\perp}\right)+\mu(\bar{b}(r, \varphi), \lambda)-\frac{1}{r}(\bar{b}(r, \varphi), \lambda)\left(\bar{b}(r, \varphi), \lambda^{\perp}\right)=0,
$$

то уравнение для $\varphi(t)$ имеет вид

$$
\varphi(t)=\varphi(0)-m \mu t+\int_{0}^{t}[-\mu+\sigma(r(s), \varphi(s))] d w(s) .
$$


428

Денисова И. Ю.

Поскольку (см. [3])

$$
\mathbf{P}\left\{\sup _{A \leqslant t \leqslant A_{1}} \frac{1}{t}\left|\int_{0}^{t}(-\mu+\sigma(r(s), \varphi(s))) d w(s)\right|>\varepsilon\right\} \leqslant \frac{c A_{1}}{\varepsilon^{2} A^{2}}
$$

тo

$$
\begin{aligned}
& \mathbf{P}\left\{\sup _{A \leqslant t} \frac{1}{t}\left|\int_{0}^{t}(-\mu+\sigma(r(s), \varphi(s))) d w(s)\right|>\varepsilon\right\} \\
& \quad \leqslant \sum_{k=0}^{\infty} \mathbf{P}\left\{\sup _{A 2^{k} \leqslant t \leqslant A 2^{k+1}} \frac{1}{t}\left|\int_{0}^{t}(-\mu+\sigma(r(s), \varphi(s))) d w(s)\right|>\varepsilon\right\} \\
& \quad \leqslant \sum_{k=0}^{\infty} \frac{c A 2^{k+1}}{\varepsilon^{2} A^{2} 2^{2 k}}=\frac{4 c}{\varepsilon^{2} A} .
\end{aligned}
$$

Величина

$$
\alpha(A)=\sup _{t \geqslant A}\left(\frac{1}{t}\right)\left|\int_{0}^{t}(-\mu+\sigma(r(s), \varphi(s))) d w(s)\right|
$$

монотонно убывает при $A \rightarrow \infty$, и, значит, существует предел $\alpha=\lim _{A \rightarrow \infty} \alpha(A)$. Тог̈да

$$
\mathbf{P}\{\alpha>\varepsilon\}=\lim _{A \rightarrow \infty} \mathbf{P}\{\alpha(A)>\varepsilon\}=0 .
$$

Поскольку $\alpha(A)$ монотонно зависит от $A$, то

$$
\mathbf{P}\left\{\lim _{t \rightarrow \infty} \frac{1}{t} \int_{0}^{t}(-\mu+\sigma(r(s), \varphi(s))) d w(s)=0\right\}=1 .
$$

Таким образом,

$$
\mathbf{P}\left\{\lim _{t \rightarrow \infty}\left(\frac{\varphi(t)}{t}\right)=-m \mu\right\}=1
$$

А если

$$
\begin{gathered}
-m \mu+\frac{1}{r}\left(\left(\bar{a}(r, \varphi), \lambda^{\perp}\right)+\mu(\bar{b}(r, \varphi), \lambda)\right)-\frac{1}{r}(\bar{b}(r, \varphi), \lambda)\left(\bar{b}(r, \varphi), \lambda^{\perp}\right)=\widehat{a}(\varphi), \\
0<-\mu+\frac{1}{r}\left(\bar{b}(r, \varphi), \lambda^{\perp}\right)=\widehat{\sigma}(\varphi)
\end{gathered}
$$

причем

$$
\left|\int_{0}^{u} \frac{\widehat{a}(v)}{\widehat{\sigma}^{2}(v)} d v\right| \leqslant c_{1},
$$

и

$$
\begin{aligned}
& \frac{1}{\varphi} \int_{0}^{\varphi} \exp \left\{-2 \int_{0}^{u} \frac{\widehat{a}(v)}{\widehat{\sigma}^{2}(v)} d v\right\} d u \rightarrow k_{1}, \quad|\varphi| \rightarrow \infty, \\
& \frac{1}{\varphi} \int_{0}^{\varphi} \exp \left\{2 \int_{0}^{u} \frac{\widehat{a}(v)}{\widehat{\sigma}^{2}(v)} d v\right\} \frac{1}{\widehat{\sigma}^{2}(u)} d u \longrightarrow k_{2}, \quad|\varphi| \rightarrow \infty, \\
& 0<k_{i}<\infty, i=1,2 \text {, то (см. [4]) } \\
& \quad P\left\{\frac{\varphi(t)}{\sqrt{t}}<y\right\} \underset{t \rightarrow \infty}{\longrightarrow} \sqrt{\frac{k_{1} k_{2}}{2 \pi}} \int_{-\infty}^{y} \exp \left\{-\frac{u^{2} k_{1} k_{2}}{2}\right\} d u .
\end{aligned}
$$

Теорема доказана. 


\section{СПИСОК ЛИТЕРАТУРЫ}

1. Бабчук В. Г. Инвариантные множества системы линейных стохастических диффузионных уравнений с постоянными коэффициентами. Препринт. Киев: ИК АН УCCP, $1980,43 \mathrm{c}$.

2. Денисова И. Ю. О достижимости и устойчивости инвариантного множества системы стохастических дифференциальных уравнений. - Укр. матем. журн., 1992, т. 44, № 4, c. 570-574.

3. Гихмая И. И., Скороход А. В. Стохастические дифференциальные уравнения. Киев: Наукова думка, 1968, 354 с.

4. Кулиячч $Г$. $Л$. Асимптотическое поведение неустойчивого решения стохастического однородного дифференциального уравнения. - Теория вероятн. и матем. статист., 1971, № 5, с. 81-87.

Поступила в редакщию

31.I.1994

(c) $1996 \mathrm{r}$.

КЛЕПЦЫНА М. Л.*

\section{ПРИНЦИП УСРЕДНЕНИЯ И ДИФФУЗИОННАЯ АІІРОКСИМАЦИЯ ДЛЯ УРАВНЕНИЙ ВОЛЬТЕРРА ${ }^{1)}$}

Рассматривается семейство случайных процессов $x^{\varepsilon}=\left(x_{t}^{\varepsilon}\right)_{t \geqslant 0}, \varepsilon \geqslant 0$, причем $x_{t}^{\varepsilon}$ - решение уравнения Вольтерра со случайными козффициентами вида

$$
x_{t}^{e}=x_{0}+\int_{0}^{t} b\left(t, x_{s}^{e}, \xi_{s / \varepsilon}\right) d s
$$

и с неслучайным начальным условием $x_{0}$, где $\xi=\left(\xi_{t}\right)_{t \in \mathbf{R}}-$ стационарный эргодический процесс. В работе находятся аппроксимации первого и второго порядков для $x^{\varepsilon}$ при $\varepsilon \rightarrow 0$ (в смысле слабой сходимости). Аппроксимацией второго порядка является решение уравнения Ито-Вольтерра.

Клюиевые слова и Фразы: приншип усреднения, диффузионная аппроксимация, сходимость по распределению, уравнение Вольтерра.

Основной целью этой работы является изучение предельного поведения семейства случайных процессов $x^{\varepsilon}=\left(x_{t}^{\varepsilon}\right)_{t \geqslant 0}$, зависящих от малого параметра $\varepsilon>0$, при $\varepsilon \rightarrow 0$. В работе изучается случай, когда $x_{t}^{\varepsilon}$ - решение уравнения Вольтерра со случайными коэффициентами, возмущенными строго стационарным эргодическим процессом. В первой части работы устанавливается принцип усреднения, аналогичный классическому принципу усреднения Боголюбова [1]. Затем находится аппроксимация второго порядка для исходного семейства процессов, точнее, устанавливается, что аппроксимацией второго порядка (диффузионной аппроксимацией) является решение стохастического уравнения Ито-Вольтерра. Следует заметить, что различными вариантами принципа усреднения и диффузионной аппроксимации занимались многие авторы (см., например, [2], [3], [4] и имеющуюся там библиографию). В отличие

*Московский государственный университет путей сообщения, кафедра прикладной математики, ул. Образцова, 15, 101475 Москва, Россия.

1) Работа выполнена при финансовой поддержке Российского фонда фундаментальных исследований (93-011-1450). 